\title{
Optimal design of plate-fin heat exchanger by combining multi- objective algorithms
}

\author{
Tariq Amin Khan \\ Wei $\mathrm{Li}^{*}$ \\ Department of Energy Engineering, Zhejiang University, Hangzhou 310027, China \\ * Corresponding author, Tel/ Fax: 86-571-87952244; E-mail:weili96@zju.edu.cn
}

\begin{abstract}
In this paper, a multi-objective optimization method is proposed which is a combination of Genetic algorithm, Differential Evolution and Adaptive Simulated Annealing algorithms. This technique is intended to generalize and improve the robustness of the three population based algorithms. In optimization problems, it is essential to keep the balance between local and global search abilities of algorithms. In the current method, DE, GA and ASA algorithms are linked in the variation stage to enrich the searching behavior and enhance the diversity of the population. The performance of the proposed DE-GA-ASA is tested against benchmark problems for multi-objectives and compared with two widely recognized vector optimizers. Next, the proposed technique is successfully implemented to optimize the design of plate-fin heat exchanger. The effectiveness of the present method is illustrated by comparing with various case studies. Some of the earlier case studies violated the constraints and/or only focused on single objective optimization. Results show that DE-GA-ASA method can be used effectively for the optimal design of plate-fin heat exchanger. Moreover, the effect of variation of fin and heat exchanger parameters on the optimal design is also investigated. Hot, cold and no-flow length of the heat exchanger, fin offset length, fin height and fin length are introduced as the optimization variables to obtain maximum heat transfer rate and minimum total annual cost. The investment cost and operating costs are independently optimized to provide a detailed investigation on the effect of fin and heat exchanger geometry parameters on their variation. Furthermore, a multi-criteria decision making method, TOPSIS is introduced for the selection of final optimal solution from the set of non-dominated solutions.
\end{abstract}

Keywords: Plate-fin heat exchanger; multi-objective optimization; Genetic algorithm; Differential Evolution; Adaptive Simulated Annealing; TOPSIS

\section{Introduction}

Heat exchangers are used to transfer thermal energy between two or more media. Various types of heat exchangers are used for different industrial applications and one of the important types is the compact heat exchanger. The advantage of these exchangers is high heat transfer area in volume unit which reduces the space, weight and ultimately the required cost and improve the energy efficiency and design process in comparison with ordinary heat exchangers [1]. The compact heat exchanger can be either plate-fin type or tube-fin type. Cross flow plate-fin heat exchangers are widely used in gas-gas applications such as cryogenics, micro-turbines, automobiles, chemical process plants, naval and aeronautical applications. The design of a PFHE is a complex task based on trial-and-error process in which geometrical and operational parameters are selected to satisfy specified requirements such as outlet temperature, heat duty and pressure drop. To increase the heat transfer area, secondary surface is connected to the primary surface. These extended surface elements are referred to as fins. Some of commonly used fins in these exchangers are plain, wavy, louver, perforated, offset strip and pin fins [2]. A typical plate-fin heat exchanger with rectangular offset strip fins shown in Fig.1 is used in this study. Since the average boundary-layer thickness decreases significantly when offset-strip fins are used, the convection coefficient increases. Therefore, these fins have higher heat transfer performance than plain flat fins [3]. However the superior thermal performance of the compact heat exchanger is at the expense of higher frictional losses (i.e. pressure drop). Therefore, the optimum design of compact heat exchanger is always required as the optimal trade-off between the increased heat transfer rate and the power consumption due to higher pressure drop within the given set of constraints. Consequently, researchers attempt to optimize thermal equipment and systems using heuristic based optimization algorithms. Moreover, finding a relation between heat transfer increment, total heat transfer area and consumption power due to high pressure drop is necessary.

There are a lot of efforts for optimization of different types of heat exchangers with various objectives and decision variables employing various methods. In addition to traditional mathematical techniques [4, 5]and artificial neural 


\begin{tabular}{|c|c|c|c|}
\hline \multicolumn{4}{|c|}{ Nomenclature } \\
\hline$A_{f}$ & flow free flow cross-sectional area $(\mathrm{m} 2)$ & $\operatorname{Pr}$ & Prandtl number (-) \\
\hline$A_{\text {tot }}$ & total heat transfer area $(\mathrm{m} 2)$ & $R e$ & Reynolds number (-) \\
\hline$b$ & fin height (m) & $S t$ & Stanton number (-) \\
\hline$c_{p}$ & specific heat $(\mathrm{J} / \mathrm{kg} \mathrm{K})$ & $t_{f}$ & fin thickness (m) \\
\hline$c$ & fin pitch (m) & $U$ & overall heat transfer coefficient $\left(\mathrm{W} / \mathrm{m}^{2} \mathrm{~K}\right)$ \\
\hline$C_{\min }$ & minimum of $C_{h}$ and $C_{c}(\mathrm{~W} / \mathrm{K})$ & $V$ & volumetric flow rate (m3/s) \\
\hline$C$ & max maximum of $\mathrm{Ch}$ and $\mathrm{Cc}(\mathrm{W} / \mathrm{K})$ & $x$ & fin length (m) \\
\hline$C^{*}$ & heat capacity rate ratio $\left(C_{\min } / C_{\max }\right)$ & \multicolumn{2}{|c|}{ Greek abbreviation } \\
\hline$D_{h}$ & hydraulic diameter (m) & $\varepsilon$ & heat exchanger effectiveness (-) \\
\hline$f$ & friction factor (-) & $h$ & compressor efficiency (-) \\
\hline$G$ & mass flux $(\mathrm{kg} / \mathrm{m} 2 \mathrm{~s})$ & $b$ & Heat transfer area per unit volume $\left(\mathrm{m}^{2} / \mathrm{m}^{3}\right)$ \\
\hline$h$ & heat transfer coefficient $\left(\mathrm{W} / \mathrm{m}^{2} \mathrm{~K}\right)$ & $m$ & viscosity (Pa. s) \\
\hline$j$ & Colburn number (-) & $n$ & $\begin{array}{l}\text { specific volume }\left(\mathrm{m}^{3} / \mathrm{kg}\right) / \text { fin } \\
\text { frequency(fins } / \mathrm{m})\end{array}$ \\
\hline$k_{f}$ & fin conductivity (W/m K) & $\Delta P$ & pressure drop $(\mathrm{Pa})$ \\
\hline$L_{c}$ & cold stream flow length $(\mathrm{m})$ & $s$ & ratio between $A_{\text {flow }}$ and $A_{\text {front }}$ \\
\hline$L_{h}$ & hot stream flow length (m) & \multicolumn{2}{|c|}{ Subscripts } \\
\hline$L_{n}$ & no-flow length (m) & $c$ & cold \\
\hline$N T U$ & number of transfer units (-) & $h$ & hot \\
\hline
\end{tabular}

network [6], many works have been conducted using evolutionary computations in design optimization of compact heat exchangers. Among these Genetic algorithm (GA) has been successfully used for optimization of plate-fin heat exchanger. Sanaye and Hajabdollahi [7] used GA to obtain the maximum effectiveness and the minimum total annual cost as two objective functions in a PFHE with offset strip fins. Xie et al. [8] minimize the total volume and total annual cost of Plate-fin type CHE with and without pressure constraint using Genetic Algorithm.

Similarly, Najafi et al. [9]optimized the total rate of heat transfer and total annual cost of the system by employing multi-objective GA. Zhe Wang et al. [10] and Zhao [11] developed a novel GA optimization model in order to effectively obtain the optimal layer pattern of multi-stream plate-fin heat exchanger. They presented few layer pattern criterion models to determine an optimal stacking pattern. They developed mentioned model by employing a genetic algorithm with binary chromosome ring representing alternatively placed hot and cold layer fluid streams. These researchers have concluded that the performance of plate fin heat exchangers in relation to heat transfer and fluid flow was effectively improved by the optimal design of the genetic algorithm layer pattern. Chyi and Hung [12] presented genetic optimization algorithm to solve the formulated multi-objective optimization problem that simultaneously minimizes the entropy generation rate and material cost of the heat sink. Varun and Siddhartha [13] have optimized a flat plate solar air heater using a genetic algorithm. They considered the thermal performance of the exchanger and optimized the exchanger considering the different system and operating parameters to obtain maximum thermal performance. Hang and Ryozo [14] obtained the optimal structural parameters of a water to water plate fin heat exchanger by using CFD and GA. In their
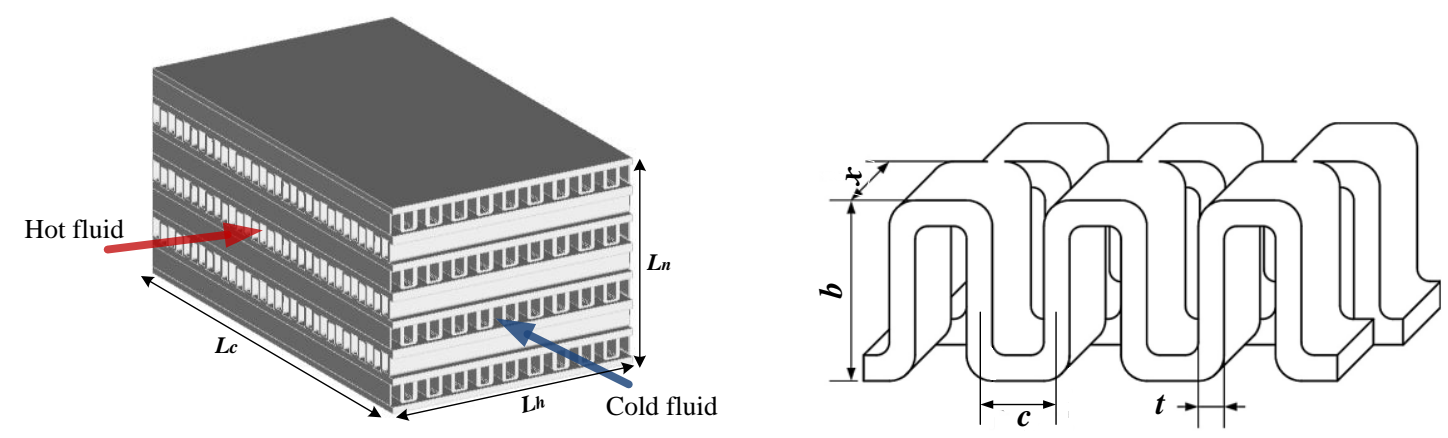

Fig.1. Schematic diagram of cross flow plate fin heat exchanger with rectangular offset strip fin.

work, the convective heat transfer coefficients of plate and fin are defined as independent parameters to obtain more 
precise results and the corresponding expressions for number of entropy generation units are deduced. In all of the above mentioned works, the Multi objective Genetic Algorithm or its extended version is used.

Apart from Genetic Algorithm, various other evolutionary algorithms in the optimization of plate fin exchangers have been developed and illustrated the need for seeking the new design methods of these equipments [15-18]. In these studies, different fitness functions such as minimization of pressure loss, weight, costs, heat transfer area are considered with various constraints and restrictions. Although many researchers have worked on the development and optimization of compact heat exchanger using new population based techniques but most of them have focused on single objective solution or violated the constraints [18-21]. Multi-objective optimization gives uncountable set of solutions called as Pareto Front. Sanaye [7], Hajabdollahi et al. [22] and Wen et al. [23] presented multi-objective optimization by implementing Non-dominated Sorting Genetic Algorithm (NSGA-II) for designing plate-fin heat exchanger. However, premature convergence and diversity in resulting population is still a challenge especially for multi-objective optimization [24, 25]. Many attempts have been undertaken to improve the performance of multi-objective optimization techniques. In this paper, a method for multi-objective optimization is used which links and interchange analogous interpreter in the search space for enhancing the diversity in population to find the best global optimal solution. It is intended to increase the robustness as it usually requires more function evaluations than a single algorithm.

This combined DE-GA-ASA method can be applied for designing plate-fin heat exchangers which is a complex task usually based on trial and error. For heat exchanger optimization, the trade-off between heat transfer and total cost is taken in to account. In general, a higher flow velocity means a higher heat transfer coefficient and hence a smaller heat transfer area and correspondingly a lower capital cost. However, higher velocity will generally lead to higher pressure drop and hence higher power consumption and correspondingly a higher power cost [26, 27]. The heat exchanger area and the pressure drop are mainly associated with capital cost (investment cost) and power cost (operating cost), respectively. In addition, in some practical applications, the plate-fin heat exchanger size is critical for compactness.

Due to the conflict nature of the thermal and economic factors, the design optimization of plate-fin heat exchanger is highly multi-objective. In this paper, Differential Evaluation (DE) [28-30], Genetic Algorithm (GA) [31, 32] and Adaptive Simulated Annealing (ASA) [33] are combined to increase the chance of global optimum points within the search space and hence a robust technique for optimization. The effectiveness of the combined DE-GA-ASA method is illustrated by comparing with the benchmark problems. It is then successfully applied for the optimization of plate-fin heat exchanger. Results of combined DE-GA-ASA are compared with earlier case studies and discussed the effectiveness of the proposed method. Then, the effect of design variables on the optimization performance of plate-fin heat exchanger is also investigated. The design variables of plate-fin heat exchanger considered in this study are fin pitch, fin height, fin offset length, hot stream flow length, cold stream flow length and no-flow length. The investment cost and operating costs are separately optimized with respect to rate of heat transfer and effects of fin and heat exchanger geometry are discussed in detail.

\section{Mathematical modeling of the exchanger}

\subsection{Thermal modeling of plat-fin heat exchanger}

A schematic of a typical cross-flow plate fin heat exchanger with offset strip fin can be seen in Fig. 1. In the heat exchangers, usually there are no external heat and work interactions and according to first law of thermodynamic, the total energy is conserved. In this study $\varepsilon-N T U$ method is applied for predicting the heat exchanger performance. The effectiveness of cross-flow heat exchanger, for both fluids unmixed is proposed as [34]:

$$
\varepsilon=1-\exp \left\{\left(1 / C^{*}\right) N T U^{0.22} \times\left(\exp \left\{-C^{*}(N T U)^{0.78}\right\}-1\right)\right\}
$$

In the mentioned equation $C^{*}$ and $N T U$, the total heat capacity ratio and number of transfer units, respectively, are defined as [2]

$$
\begin{aligned}
& N T U_{\text {max }}=\frac{U A_{t o t}}{C_{\text {min }}} \\
& C^{*}=\frac{C_{\text {min }}}{C_{\text {max }}}
\end{aligned}
$$

where $A_{\text {tot }}$ is total heat transfer area and $U$ is the overall heat transfer coefficient. The overall heat transfer coefficient is a function of convection heat transfer resistance in each side (cold and hot) as well as conduction heat transfer resistance. Usually a very thin plate is used between the fins in each side and as a result the conduction resistance is negligible 
compared with the convection resistance. Based on the mentioned assumption, the overall heat transfer coefficient is proposed as:

$$
\begin{aligned}
& U=\frac{1}{\frac{1}{\left(h \eta_{s}\right)_{c}}+\frac{1}{\frac{A_{t o t, h}}{A_{t o t, c}}\left(h \eta_{s}\right)_{h}}} \\
& A_{t o t}=\left(\beta V_{p}\right)_{c}+\left(\beta V_{p}\right)_{h}
\end{aligned}
$$

$\beta$ is the heat transfer surface area per unit volume defined as:

$\beta=\frac{A_{c e l l}}{V_{\text {cell }}}=\frac{2\left(b-t_{f}\right) x+2\left(c-t_{f}\right) x+x\left(b-t_{f}\right) t_{f}+c t_{f}}{b c x}$

Where $b, t_{f}, x$ and $c$ are height, thickness, length and pitch of the fin, respectively.

The rate of heat transfer rate is calculated as:

$Q=\varepsilon C_{\min }\left(T_{\mathrm{h}, 1}-T_{\mathrm{c}, 1}\right)$

The volume between plates for hot and cold stream sides $\left(V_{p}\right)$ of the heat exchanger is defined as:

$V_{p}=L_{c} L_{h} b N$

By assuming there are $\mathrm{N}$ passages for hot side and $\mathrm{N}+1$ passages for the cold side, therefore:

$N=\frac{L_{n}-b_{c}+2 t_{w}}{b_{h}+b_{c}+2 t_{w}}$

where $t_{w}$ is the thickness of plate. Moreover, the overall surface efficiency $\left(\eta_{s}\right)$ is defined as [2]:

$\eta_{s}=1-\frac{A_{f}}{A_{\text {cell }}}\left(1-\eta_{f}\right)$

Where $A_{f}$, the fin heat transfer area and $\eta_{f}$, the fin efficiency can be calculated as [34]:

$A_{f}=2\left(b-t_{f}\right) x+2\left(b-2 t_{f}\right) t_{f}+c t_{f}$

$\eta_{f}=\tan (m l) / m l$

Where $m=\sqrt{2 h /\left(k_{f} t_{f}\right)}$

and $l=b / 2$

There are many correlations for evaluation of Colburn factor $j$ and Fanning factor $f$ for offset strip fin. Eqs. (13) and (14) are the correlation proposed by Manglik and Bergles [35] for rectangular offset strip fin cores are used in this work which is given by:

$$
\begin{aligned}
j= & 0.6522(\mathrm{Re})^{-0.5403}(\alpha)^{-0.1541}(\delta)^{0.1499}(\gamma)-0.0678 \\
& \times\left[1+5.269 \times 10^{-5}(\mathrm{Re})^{1.34}(\alpha)^{0.504}(\delta)^{0.456}(\gamma)^{-1.055}\right]^{0.1} \\
f= & 9.6243(\operatorname{Re})^{-0.7422}(\alpha)^{-0.1856}(\delta)^{0.3053}(\gamma)^{-0.2659} \\
& \times\left[1+7.669 \times 10^{-8}(\operatorname{Re})^{4.429}(\alpha)^{0.920}(\delta)^{3.767}(\gamma)^{0.236}\right]^{0.1}
\end{aligned}
$$


The above correlations are accurate within $\pm 20 \%$ and are valid for $120<\operatorname{Re}<10^{4}, 0.134<\alpha<0.997,0.012<\delta<0.048$ and $0.041<\gamma<0.121$, where:

$\alpha=c / b, \delta=t_{f} / x, \gamma=t_{f} / c$

In addition, $R e$ is Reynolds number as a function of mass flux, hydraulic diameter and viscosity defined as [2]:

$\operatorname{Re}=\frac{G D_{h}}{\mu}$

$D_{h}=\frac{4\left(c-t_{f}\right)\left(b-t_{f}\right) x}{2\left(\left(c-t_{f}\right) x+\left(b-t_{f}\right) x+\left(b-t_{f}\right) t_{f}\right)+\left(c-t_{f}\right) t_{f}-t_{f}^{2}}$

$G=\frac{m}{A_{\text {flow }}}$

Where Aflow is free flow cross-sectional area defined as:

$A_{\text {flow }}=\frac{D_{h} A_{\text {tot }}}{4 L}$

The convective heat transfer coefficient $h$ and the Stanton number $S t$ are defined as:

$h=S t G c_{p}$

$S t=j / \operatorname{Pr}^{2 / 3}$

where $\mathrm{P}_{\mathrm{r}}$ is the Prandtl number. Furthermore, the pressure drop by neglecting the inlet and outlet pressure drop in each side is estimated as [2]:

$\Delta p_{a}=\frac{2 f_{a} L_{a} G_{a}^{2}}{\rho_{a} D_{h, a}}$

$\Delta p_{b}=\frac{2 f_{b} L_{b} G_{b}^{2}}{\rho_{b} D_{h, \mathrm{~b}}}$

\subsection{Economic modeling}

The cost of heat exchanger, including investment cost and operating cost are mathematically modeled as below [8]:

$C_{i n}=A_{f} \cdot C_{A} \cdot A_{t o t}^{n_{1}}$

$C_{o p}=\left[k_{e l} \tau \frac{\Delta P \cdot m}{\eta \cdot \rho}\right]_{a}+\left[k_{e l} \tau \frac{\Delta P \cdot m}{\eta \cdot \rho}\right]_{b}$

In the above, $C_{A}$ and $n_{l}$ are cost per unit surface area and numeric exponent respectively. Moreover, $k_{e l}, \tau$ and $\eta$ are the electricity price, hours of operation and compressor efficiency respectively. In Eq. (22) $A_{f}$ is the annual coefficient factor that given as:

$A_{f}=\frac{r}{1-(1+r)^{-y}}$

where $\mathrm{r}$ and $\mathrm{y}$ are interest rate and depreciation time, respectively. The parameters required for cost calculation of the heat exchanger are listed in Table 1. 


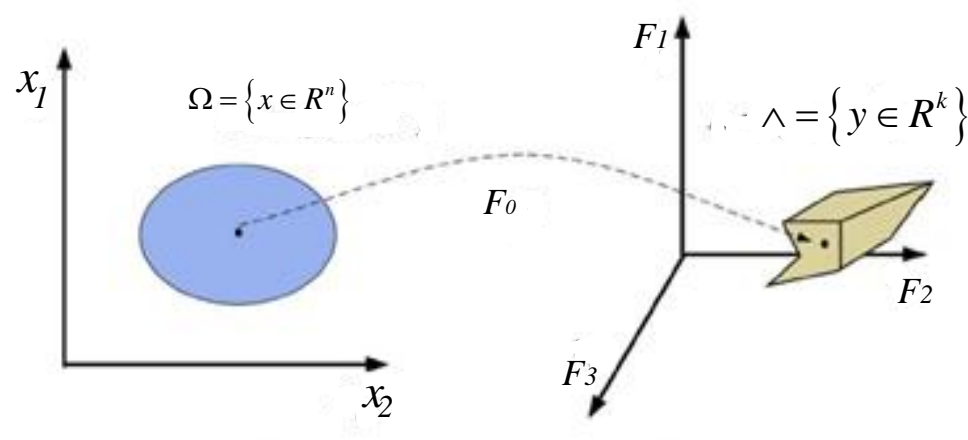

Design variable space.

,Objective function space

Fig. 2. A schematic view of multi-objective mapping, $n=2, m=0, k=3[28]$

\section{Multi-objective optimization}

There are indeed rare problems in the real world which only deal with a single objective. The optimal design of platefin heat exchanger is complex which involves multi-objectives and relies on many parameters. In this study a multiobjective optimization method is proposed and implemented to achieve the optimization target.

Multi-objective optimization, which is also called multi-criteria optimization or vector optimization is defined as finding a vector of decision variables satisfying constraints to give acceptable values to all objective functions. It gives the set of several solutions because of conflicting variables and is called Pareto front. Hence, a trade- off between two or more objective functions is needed to make the optimal decision. A reasonable solution to a multi-objective problem is to investigate a set of solutions, each of which satisfies the objectives at an acceptable level without being dominated by any other solution $[32,36]$. As the objective functions are non-dominated compared with each other, the optimal Pareto set gives the better and worst values by moving along the series of points.

Since objectives can be either minimized or maximized, the general form of multi-objective optimization problem is given as:

$$
\left.\begin{array}{rlr}
\text { Minimize/Maximize } & f_{m}(\mathrm{x}), & m=1,2, \ldots \ldots, M ; \\
\text { subject to } & g_{j}(\mathrm{x}) \geq 0, & j=1,2, \ldots \ldots, J ; \\
& h_{k}(\mathrm{x})=0, & k=1,2, \ldots \ldots, K ; \\
& x_{i}^{(L)} \leq x_{i} \leq x_{i}^{(U)}, & i=1,2, \ldots \ldots, n .
\end{array}\right\}
$$

where a solution $\mathrm{x} \in \mathrm{R}^{\mathrm{n}}$ is a vector of $\mathrm{n}$ decision variables:

$\mathrm{x}=\left(x_{1}, x_{2}, \ldots . ., x_{n}\right)^{T}$

The lower bound $x_{i}^{(L)}$ and the upper bound $x_{i}^{(U)}$ give the range of decision variables. $g_{j}(\mathrm{x})$ and $h_{k}(\mathrm{x})$ associate with constraint category where $J$ and $K$ denote the number of inequality and equality constraints, respectively. Vector $f$ defines $m$ objective functions as:

$$
f(x)=z=\left(z_{1}, z_{2}, \ldots \ldots, z_{M}\right)^{T}
$$

The multi-objective optimization function, $f: \Omega \rightarrow \Lambda$ maps decision variables $\left(x=x_{1}, x_{2}, \ldots \ldots, x_{n}\right)$ to vectors $(y=$ $\left.a_{1}, a_{2}, \ldots . . a_{k}\right)$ as shown in Fig. 2. This mapping is solely dependent on the objective functions and constraints under consideration for multi-objective optimization. As discussed earlier, premature convergence is a major challenge in solving multi-objective optimization problems which means that the population is trapped in the local minima and nondominance is not guaranteed for convergence to the true Pareto front. In this study, three multi-objective optimizers i.e., Genetic Algorithm, Differential evolution, and Adaptive Simulated Annealing are combined to increase the chance of finding global optimum. The three algorithms are briefly discussed in the following section.

\subsection{Differential Evolution}

Differential evolution (DE) is a simple yet powerful evolutionary algorithm proposed by Storn and Price [37]. DE has been consistently ranked as one of the best search algorithm for solving global optimization problems in several case 
studies [38]. Most commonly, it consists of three operations: mutation, crossover, selection. DE is different from traditional evolutionary algorithms (EAs) in the sense that it perturbs the current generation population members with the scaled differences of randomly selected and distinct population members. It uses a non-uniform crossover where offspring vectors are inherited in unequal proportions from parent vectors and promotes a stable series of solutions by fast convergence towards the Pareto front [28-30]. The overall structure of DE resembles most of that others population based searches. The parallel version of DE holds a population of $N P$, D-dimensional, real valued vectors. In each generation, $N P$ competitions are held to determine next generation. A pair of vectors $\left(X_{a}, X_{b}\right)$ are chosen randomly and their weighted differential $\left(X_{a}-X_{b}\right)$ is used to perturb another randomly chosen vector $X_{c}$, i.e., $X_{c}^{\prime}=X_{c}+F\left(X_{a}-X_{b}\right)$, where $F$ is the scaling factor. After producing the trial vector $X_{t}$ from crossover, it is compared with the target vector and the vector that has lowest cost of the two would survive for next generation [37].

\subsection{Genetic Algorithm:}

Genetic Algorithm (GA) is a stochastic numerical search method which was first conceived by Holland [39]. Fonseca proposed a modified form of Schaffer's technique called Multi-objective Genetic Algorithm (MOGA) which explicitly uses Pareto-based ranking and niching techniques together to encourage the search towards true Pareto front while maintaining diversity in the population $[31,32]$. GA operates on a population of potential solution vectors $(x \in \mathrm{X})$ in each iteration (i.e. generation). The initial population is usually randomly generated and the parents are selected based on their fitness values in order to maintain diversity of individual. Since the individuals with higher fitness have more chance for being selected for reproduction, consequently, the individuals in the next generation will possess higher fitness. Therefore, using crossover operator iteratively leads to convergence to general good solution. If the individual $x_{i}$ in generation $t$ is dominated by $p_{i}^{(t)}$ individuals in the current generation then individual $x_{i}$ is assigned a ranked by the rule; $\operatorname{rank}\left(x_{i}, t\right)=1+p_{i}^{(t)}$ The mutation operator creates a new solution by random change in an individual with a probability $P_{m u t}$. It insures that the probability of searching any new string will never be zero and acting as safety net to recover good genetic material which may be lost through the action of selection and crossover.

\subsection{Adaptive Simulated Annealing}

The simulated annealing is a meta-heuristic method which solves combinatorial optimization problems and is based on the physical phenomenon of annealing [33, 40]. It uses a generalized form of Metropolitan criterion [40] to have a better chance to obtain a global minimum and escape from being trapped in a local minimum energy state. Adaptive simulated annealing (ASA) [33] relies on randomly importance-sampling in the parameter space. It is advantageous by the fact that the parameter space can be sampled much more efficiently than other previously simulated annealing algorithms. ASA generates a random perturbation that displaces a "particle"(moving the configuration of $X_{0}$ to other configuration $\left.X_{i}\right)$. If the new configuration is better than the current one $\left(E\left(X_{i}\right)<E\left(X_{0}\right)\right)$, it is adopted as a new current solution. However, if the new configuration turns out to be worse than current solution $\left(E\left(X_{i}\right) \geq E\left(X_{0}\right)\right)$, the move is either accepted with some acceptance probability, or rejected. By giving a chance to move to inferior solutions make the algorithm capable for escaping from local minima. The energy state $\left(E\left(X_{0}\right)\right)$ is equivalent to the objective function where $X_{0}$ is a design variable. Finding a low energy configuration is equivalent to finding a near optimal solution. The function that gives an acceptance probability of a bad solution is:

\section{$P=\operatorname{Exp}(-(F[$ candidate solution $]-F[$ current solution $]) / T)$}

Where $F$ is the evaluation function and $t$ is the control parameter called temperature. If the initial value of $T$ is very small, the solution cannot escape from local proximity of the initial solution and approximates to a local optimal solution in this region. On the other hand, a very high initial value of $T$ leads to extended search before starting to intensify on some good regions. Similarly, the cooling schedule consists of a set of decreasing temperatures and the amount of time spends at each temperature.

In the ASA, the temperature is adjusted dynamically according to the knowledge obtained in the past search. Such an approach decreases the dependence of the algorithm on the initial temperature and cooling schedule, which is actually a great advantage of the adaptive cooling schedule.

The Pseudo codes of DE, GA and ASA are given in Appendix I.

\subsection{Combined DE-GA-ASA}

As discussed earlier, Differential evolution (DE), Genetic algorithms (GA) and Adaptive simulated annealing (ASA) are powerful stochastic search techniques for solving global optimization problems. However, the control parameters and learning strategies involved are highly dependent on the problem under consideration. Even adapting the control parameters in successive generations in DE does not guarantee for optimal solution because some parameters are still fixed. Due to slow convergence, the accuracy can decrease by trapping in local optima [38]. 


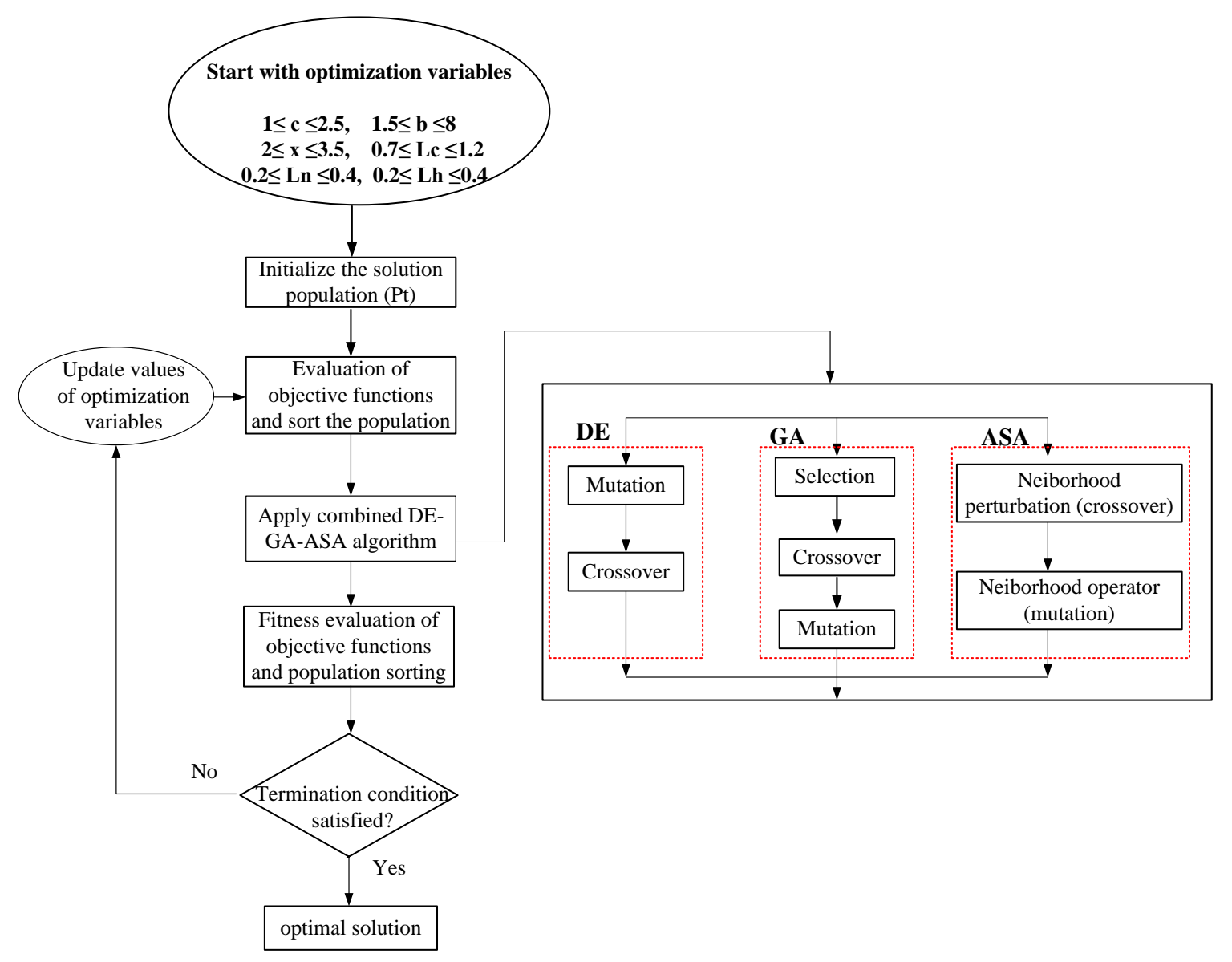

Fig. 3. The flow-chart of DE-GA-ASA method

Hence, despite their application to solve wide range of design problems, they also suffer from premature convergence and insufficient diversity in either earlier or later stages. For example, initially, the convergence behavior of GA is more rapid but then it becomes very difficult to obtain further improvement. While ASA does not converge as fast as GA in the initial phase but it is able to obtain improvement in late phase of the process. This is probably due to the insufficient dimensional searching strengths, especially for problems with many decision parameters. Hence, each algorithm though powerful in their own way also has shortcomings. It is well known that there is no single universal optimizer that can be applied to either binary or continuous optimization [41]. Thus, there is a need to investigate a universal optimizer for the design investigation of complex models such as compact heat exchangers.

For this goal, the current work proposed a combined approach of DE, GA and ASA algorithm to improve the global search ability and convergence. In the proposed algorithm, a collaboration-based relation [42] exist among DE, GA and ASA where they exchange or share accumulated information while their own basic operating steps are maintained to generate new sampling points in the search space. The population is randomly initialized and the objective function is obtained for each individual. The whole population is divided into three sub-populations which are randomly extracted from it. Each individual of these sub-populations is a candidate solution of the problem. In the case of GA, the selection is based on their feasibility to form the mating pool. On the other hand, no mating pool is created corresponding to DE and ASA. Combining DE, GA and ASA is implemented in the variation operation step in every generation. The iterative procedure of the proposed DE-GA-ASA method is illustrated in Fig. 3.

In GA, the crossover operator probabilistically works by selecting two parents and they are swapped at their gene level to produce two offspring. The mutation operator probabilistically works by selecting two units within an individual and swap them [32].

DE creates a mutant individual $\left(X_{i, g+1}\right)$ from $i$ th individual at generation $g$. The mutation operator is given by;

$X_{i, g+1}=X_{r, g}+F_{1}\left(X_{r 1, g}-X_{r 2, g}\right)$ 
Where $r_{1}, r_{2} \in\{1,2,3 \ldots, N P\}$ which are mutually exclusive and randomly chosen indices and also different from index $i$. Once the mutation phase is over, the mutant individual $\left(X_{i, g+l}\right)$ is mixed with the parent individual $\left(X_{r, g}\right)$ to produce a trial individual $V_{j, i, g+l}$. A binary crossover is implemented where the trial individual is inserted either from the mutant individual or parent individual according to the following condition:

$u_{j, i, g+1}=\left\{\begin{array}{lc}v_{j, i, g+1} & \text { if } \quad \text { rand } \leq C r \vee j=k \\ x_{j, i, g} & \text { otherwise }\end{array}\right.$

Where $j, k \in\{1,2, \ldots . ., D\}$ ( $D$ being the number of decision variables); $k$ is a random parameter chosen once for each $i$ and rand $_{j} \in[0,1]$. $\mathrm{Cr}$ is the crossover constant takes values in the range $[0,1]$.

In ASA, a parameter $a_{\beta+1}^{i}$ in dimension $i$ generated by perturbation at annealing time $t$ with the random variable $y_{i}$ is:

$a_{\beta+1}^{i}=a_{\beta}^{i}+y^{i}\left(B_{i}-A_{i}\right), y^{i} \in[-1,1]$

Where $a_{\beta}^{i} \in\left[A_{i}, B_{i}\right]$ and $y^{i} \in[-1,1]$. The new parameters $a_{\beta+1}^{i}$ are generated from the old parameter $a_{\beta}^{i}$ by uniform distribution until a set of D-dimensional parameters are obtained satisfying the range of constraints [33]. The Metropolice criteria [40] mutate the parameters to maintain diversity by escaping from local minimum energy state (local optimal solution). After obtaining the sampling points (individuals) from DE-GA-ASA, these offspring individuals in the search space undergo fitness evaluation. The parent population and offspring population are ranked based on their fitness to create the population of the next generation. Hence, the three optimizers work independently and at every generation, they share new information with each other. Thus, the cooperation among DE, GA and ASA exists in the search space to obtain the optimum solution.

\section{Design of Plate fin heat exchanger using combined DE-GA-ASA algorithm}

In this study the combined DE-GA-ASA optimization method is applied to investigate the optimal design of plate-fin heat exchanger. The optimum selection and comprehensive performance evaluation is illustrated in Fig. 3 . The steps involved are described as follows:

i. Initialize the DE, GA and ASA parameters (The design variables and objective functions are specified).

ii. The initial position is generated (i.e., fin height, length, pitch, heat exchanger hot stream length, cold stream length and no-flow length) which are uniformly and randomly distributed within their corresponding ranges (max and min limits) satisfy the constraints and restrictions discussed in section 2.

iii. The initial population (heat transfer rate, total annual cost) is generated which are possible solution of the problem. The solutions are sorted and ranked based on the corresponding objectives. The number of feasible solutions and the best infeasible solutions are selected based on the population size. The population size selected in this study is 800 .

iv. DE, GA and ASA are utilized to form new sets for the design variables to generate updated solutions (three subpopulations).

v. The three sub-populations (solution sets) are unified to form a single population.

vi. Feasible solutions are selected to generate the population of next generation.

vii. The optimization process is terminated when the stopping criteria is fulfilled.

\section{Decision making criteria}

In multi-objective optimization, there exists multiple non-dominated points as close as possible to the true Paretooptimal front with a wide trade off among the objectives. The final optimal solution is then obtained from the available solutions of the Pareto front. In this paper, the Technique for Order of Preference by Similarity to Ideal Solution (TOPSIS) [43] is introduced for choosing the final solution.

TOPSIS is a multi-criteria decision analysis method, which is used to rank the alternatives of the obtained Pareto solutions. In TOPSIS the distance from the target alternative $i$ of the $j_{t h}$ objective to the best $\left(d_{i b}\right)$ and worst $\left(d_{i w}\right)$ conditions are calculated as: 
Table 1

Mean (first row) and Variance (second row) of the convergence metric

\begin{tabular}{ccccc}
\hline Algorithm & FON & ZDT3 & ZDT4 & ZDT6 \\
\hline \multirow{2}{*}{ NSGAII (Real) } & 0.001931 & 0.1145 & 0.513053 & 0.296364 \\
\cline { 2 - 5 } & 0 & 0.00794 & 0.11846 & 0.013135 \\
\hline \multirow{2}{*}{ NSGAII (binary) } & 0.002571 & 0.043411 & 3.227636 & 7.806798 \\
\cline { 2 - 5 } & 0 & 0.000042 & 7.30763 & 0.001667 \\
\hline \multirow{2}{*}{ PAES } & 0.151263 & 0.023872 & 0.854816 & 0.085469 \\
\cline { 2 - 5 } & 0.000905 & 0.00001 & 0.527238 & 0.006664 \\
\hline \multirow{2}{*}{ DE-GA-ASA } & 0.01022 & 0.04851 & 0.320021 & 0.009325 \\
\cline { 2 - 5 } & 0.000078 & 0.00063 & 0.065242 & 0.00143 \\
\hline
\end{tabular}

Table 2

Mean (first row) and Variance (second row) of the diversity metric

\begin{tabular}{ccccc}
\hline Algorithm & FON & ZDT3 & ZDT4 & ZDT6 \\
\hline \multirow{2}{*}{ NSGAII (Real) } & 0.378065 & 0.73854 & 0.702612 & 0.668025 \\
\cline { 2 - 5 } & 0.000639 & 0.019706 & 0.064648 & 0.009923 \\
\hline \multirow{2}{*}{ NSGAII (binary) } & 0.395131 & 0.575606 & 0.479475 & 0.644477 \\
\cline { 2 - 5 } & 0.001314 & 0.005078 & 0.009841 & 0.035042 \\
\hline \multirow{2}{*}{ PAES } & 1.162528 & 0.78992 & 0.870458 & 1.153052 \\
\cline { 2 - 5 } & 0.008945 & 0.001653 & 0.101399 & 0.003916 \\
\hline \multirow{2}{*}{ DE-GA-ASA } & 0.321388 & 0.498816 & 0.623371 & 0.372114 \\
\cline { 2 - 5 } & 0.003251 & 0.005453 & 0.036455 & 0.007231 \\
\hline
\end{tabular}

$\left.\begin{array}{l}d_{i b}=\sqrt{\sum_{j=1}^{n}\left(t_{i j}-t_{b j}\right)^{2}}, \quad i=1,2, \ldots \ldots, m \\ d_{i w}=\sqrt{\sum_{j=1}^{n}\left(t_{i j}-t_{w j}\right)^{2}}, \quad i=1,2, \ldots \ldots, m\end{array}\right\}$

And the relative closeness to the ideal solution $\left(s_{i w}\right)$ is calculated as:

$s_{i w}=d_{i w} /\left(d_{i w}+d_{i b}\right), \quad 0 \leq s_{i w} \leq 1$

The best compromised solution is one whose $s_{i w}$ is closest to 1 .

\section{Validation}

In order to validate the performance of the proposed algorithm, four benchmark problems, FON, ZDT3, ZDT4, ZDT6 [44] with different Pareto optimal fronts are selected. These functions have two objectives and no constraints. The nature of the Pareto front of these problems is different. The Pareto front of FON and ZDT4 are non-convex; ZDT3 is convex and disconnected while ZDT6 has non-convex and non-uniform Pareto front.

The numerical tests are compared with the NSGAII and PAES [44]. To obtain the stochastic performance metrics, the algorithm is run 20 times. The performance is measured by the divergence metric and diversity metric as suggested by Deb K. [44]. The divergence metric measures the extent of convergence to a known set of Pareto optimal solution. The smaller this metric, the better is the convergence towards the true Pareto front. The diversity metric measures the spread 

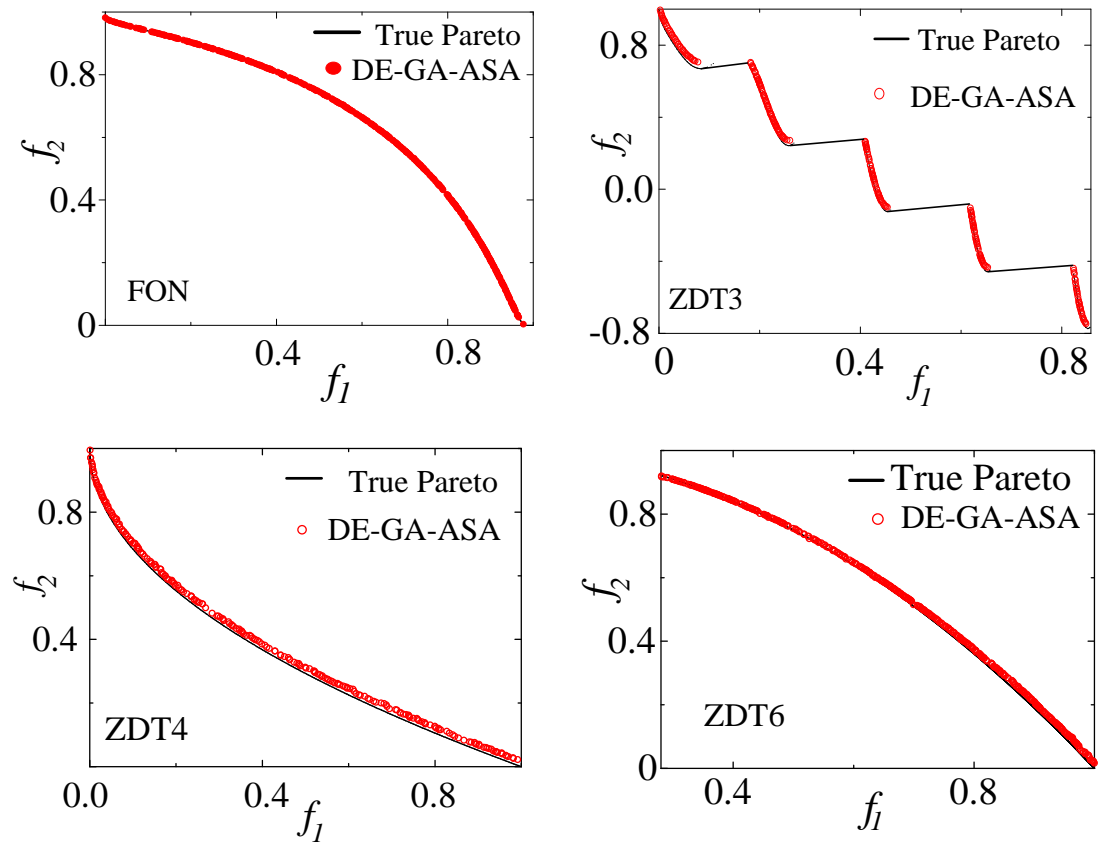

Fig. 4. Searched Pareto front by DE-GA-SA

among the obtained solutions; the smaller is this metric the more uniform distribution of the searched solutions. The corresponding results from the present DE-GA-ASA method are presented in Table 1 and Table 2 along with NSGAII and PAES. From Table 1, it can be observed that for FON and ZDT3, DE-GA-ASA and NSGAII (binary) has almost same convergence performance, while DE-GA-ASA outperformed for ZDT4 and ZDT6. Similarly, the diversity performance of DE-GA-ASA is better than both PAES and NSGAII (binary) except ZDT4. The obtained of Pareto fronts of DE-GA-ASA are shown in Fig. 4.

\section{Case study}

The optimum design parameters of the plate-fin heat exchanger are obtained for a gas furnace in a tile factory located in northwest of Kerman city, the biggest province in Iran. Furnace temperature is about $380 \mathrm{~K}$ in the initial stages and $1200 \mathrm{~K}$ in the last stages. The hot gas (hot stream) exits from the middle stages of furnace with $1.45 \mathrm{~kg} / \mathrm{s}$ mass flow rate and passes through the heat exchanger at $620 \mathrm{~K}$. The fresh air (cold stream) passes through the exchanger at $315 \mathrm{~K}$ with $1.35 \mathrm{~kg} / \mathrm{s}$ mass flow rate. Operating conditions and the other constant values are listed in Table 6. The thermophysical properties of air such as Prandtl number, viscosity and specific heat are considered as a function of temperature. The design parameters and their range of variation used in this study are listed in Table 7.

\section{Objective functions, design parameters and constraints}

In this paper, obtaining maximum heat transfer rate, investment cost, operating cost and the total annual cost are considered as objective functions. A minimum of function $-f$ is a maximum of function $f$. That is, for converting the minimization problem to maximization problem, it is enough to multiply the minus in the objective function. Furthermore, fin pitch $(c)$, fin height $(b)$, fin offset length $(x)$, cold stream flow length $\left(L_{c}\right)$, hot stream flow length $\left(L_{h}\right)$ and no-flow length $\left(L_{n}\right)$ are considered as six design parameters.

The constraints are also introduced as follows:

$0.134<\alpha<0.997$

$0.012<\delta<0.048$

$0.041<\gamma<0.121$

$120<\operatorname{Re}<10^{4}$ 
Table 3

Optimal parameters for minimum $A_{t o t}$ and $f_{x}$

\begin{tabular}{ccc}
\hline & Minimum $A_{\text {tot }}$ & Minimum $f_{x}$ \\
\hline$L_{h}(\mathrm{~m})$ & 0.26 & 1 \\
$L_{c}(\mathrm{~m})$ & 0.186 & 1 \\
$b(\mathrm{~mm})$ & 6.81 & 10 \\
$t_{f}(\mathrm{~mm})$ & 0.1 & 0.2 \\
$n$ & 1000 & 201 \\
$x(\mathrm{~mm})$ & 2.14 & 10 \\
$N_{a}$ & 79 & 72 \\
Re $_{c}$ & 507.37 & 391.36 \\
Re $_{h}$ & 1007 & 554.92 \\
$\Delta P_{h}(\mathrm{Kpa})$ & 9.46 & 0.2885 \\
$\Delta P_{c}(\mathrm{Kpa})$ & 7.9717 & 0.2055 \\
$f_{a}$ & 0.09723 & 0.09281 \\
$f_{b}$ & 0.06338 & 0.071685 \\
$j_{a}$ & 0.02293 & 0.0209 \\
$j_{b}$ & 0.0162 & 0.01763 \\
$\varepsilon$ & 0.82058 & 0.82058 \\
$Q(\mathrm{KW})$ & 1069.8 & 1069.8 \\
Objective $f(x)$ & 1.992 & $\mathbf{0 . 0 5 6 0 5}$ \\
Objective: $A_{\text {tot }}$ & $\mathbf{1 1 0 . 8 8}$ & 706.36 \\
\hline & &
\end{tabular}

\section{Result and discussion}

The performance of proposed DE-GA-ASA method for plate-fin heat exchanger is investigated by comparing the results with previous works.

For comparison purpose, constraint on the maximum pressure drop and total heat transfer rate $(Q)$, are also imposed (i.e., $Q=1069.8\left(\mathrm{KW}, \Delta P_{h} \leq 9.5(\mathrm{KPa}), \Delta P_{c} \leq 8.0(\mathrm{KPa})\right)$. The details can be found in [21]. The results of minimum heat transfer area $\left(A_{h t}\right)$ and pressure drop factor $\left(f_{x}\right)$ along with detail parameters are given in Table 3 and the comparison to other case studies are given in Table 4 and Table 5. It can be seen from Table 4 that the result of IHS method is better than other techniques; however, it violate some constraints. Similarly, for the minimum pressure drop factor $\left(f_{x}\right)$ hybrid GA and IHS methods violates the constraints which are highlighted [21]. Overall, the result of BBO algorithm and DEGA-ASA method are within boundary constraints. Nevertheless, the BBO algorithm has better results than all other approaches; however, it is not investigated in detail for multi-objective optimization.

\subsection{Optimal parameters investigation}

In previous section, the feasibility of the proposed method is illustrated. In this section, the thermal and economic design of plate-fin heat exchanger is discussed by considering the design parameters and the operating conditions given in Table 6 and Table 7. The total annual cost is divided in to investment cost and operating cost and optimized separately. In the first scenario optimization is carried out for maximum rate of heat transfer and minimum $T A C$ as shown in Fig 5. The result is a set of optimal solutions each of which is a trade-off between the objective functions. For the present case study, it can be seen that the heat transfer rate varies from $3.3588 \times 10^{5}(\mathrm{~W})$ to $3.9774 \times 10^{5}$ while the TAC ranges from 593.88 (\$/yr) to 3794.4 (\$/yr).

Furthermore, the investment cost $\left(C_{i n}\right)$ and operating cost $\left(C_{o p}\right)$ are also optimized independently with respect to the rate of heat transfer as shown in Fig. 6 and 7. The rate of heat transfer increases with increasing the heat transfer area but at the same time investment cost also increases. The operating cost depends on the pressure drop which generally increases with increasing the heat transfer area. 
Table 4

Results and values of constraints for Minimum $A_{h t}$

\begin{tabular}{cccccc}
\hline & $\begin{array}{c}\text { Preliminary } \\
\text { design }\end{array}$ & Hybrid GA & IHS & BBO & DE-GA-ASA \\
\hline$\alpha$ & 0.493 & 0.155 & 0.136 & 0.134 & 0.1341 \\
$\delta$ & 0.031 & 0.048 & 0.058 & 0.048 & 0.0467 \\
$\gamma$ & 0.084 & 0.111 & 0.111 & 0.121 & 0.111 \\
$Q(\mathrm{KW})$ & - & - & - & 1069.8 & 1069.8 \\
$A_{h t}\left(\mathrm{~m}^{2}\right)$ & 142.75 & 112.69 & 109.62 & 110.02 & 110.88 \\
\hline
\end{tabular}

Table 5

Results and values of constraints for Minimum $f_{x}$

\begin{tabular}{ccccc}
\hline & $\begin{array}{c}\text { Hybrid } \\
\text { GA }\end{array}$ & HIS & BBO & DE-GA-ASA \\
\hline$\alpha$ & 0.409 & 0.468 & 0.492 & 0.4873 \\
$\delta$ & 0.01 & 0.01 & 0.02 & 0.0204 \\
$\gamma$ & 0.025 & 0.021 & 0.041 & 0.0419 \\
$L_{n}(\mathrm{~m})$ & 1.437 & 1.437 & 1.5 & 1.5 \\
$Q(\mathrm{KW})$ & 1085.95 & 1068.67 & 1069.8 & 1069.8 \\
$f_{x}$ & 0.0619 & 0.054 & 0.052 & 0.05605 \\
\hline
\end{tabular}

Thus, increasing the heat transfer area increases the pressure drop (Eq. (18)) and operating cost along with the investment cost. To investigate the effect of design variables on the costs of plate-fin heat exchangers in detail are discussed in next section.

\subsection{Optimum parameters}

Two kinds of parameters including the fin related parameters (pitch, height, length) and heat exchanger parameters (hot stream length, cold stream length, no-flow length) are investigated. The distribution of variables for the optimal points on Pareto front in Fig. 5, 6 and Fig.7 are shown in Fig. 8(a-f). The investment cost $\left(C_{i n}\right)$ and operating cost $\left(C_{o p}\right)$ are optimized to investigate the difference in trend between minimizing the heat transfer area and the operating cost Increasing costs by varying the decision variables are also shown by arrows in Fig. 8( $a-f)$.

\subsubsection{Fin height}

The fin height values affect the optimal points on Pareto front as the fin height values cover its whole domain for

\section{Table 6}

Operating parameters of the PFHE

\begin{tabular}{lr} 
Mass flow rate of hot flow $(\mathrm{kg} / \mathrm{s})$ & 1.45 \\
Mass flow rate of cold flow $(\mathrm{kg} / \mathrm{s})$ & 1.35 \\
Inlet hot temperature $(\mathrm{K})$ & 620 \\
Inlet cold temperature $(\mathrm{K})$ & 315 \\
Inlet pressure (hot side) $(\mathrm{kPa})$ & 180 \\
Inlet pressure (cold side) $(\mathrm{kPa})$ & 120 \\
$C_{A}(\$ / \mathrm{m})$ & 90 \\
$n_{l}$ & 0.6 \\
Unit price of elecricity $(\$ / \mathrm{MWh})$ & 20 \\
interest rate & 0.1 \\
Pump efficiency & 0.6 \\
Life time (years) & 10 \\
\hline
\end{tabular}

\section{Table 7}

The Design parameters and their range of variation

\begin{tabular}{lcc}
\hline Variables & From & To \\
\hline Fin pitch (mm) & 1 & 2.5 \\
Fin height (mm) & 1.5 & 8 \\
Fin length (mm) & 2 & 3.5 \\
Hot stream flow length (m) & 0.2 & 0.4 \\
Cold stream flow length (m) & 0.7 & 1.2 \\
No-flow length (m) & 0.2 & 0.4 \\
\hline
\end{tabular}




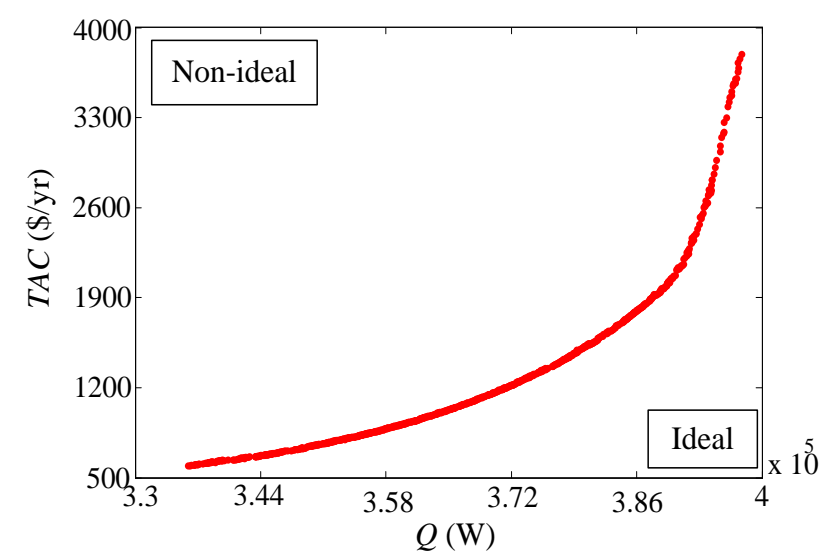

Fig. 5. Rate of heat transfer $(Q)$ vs. TAC Pareto front in the objective space

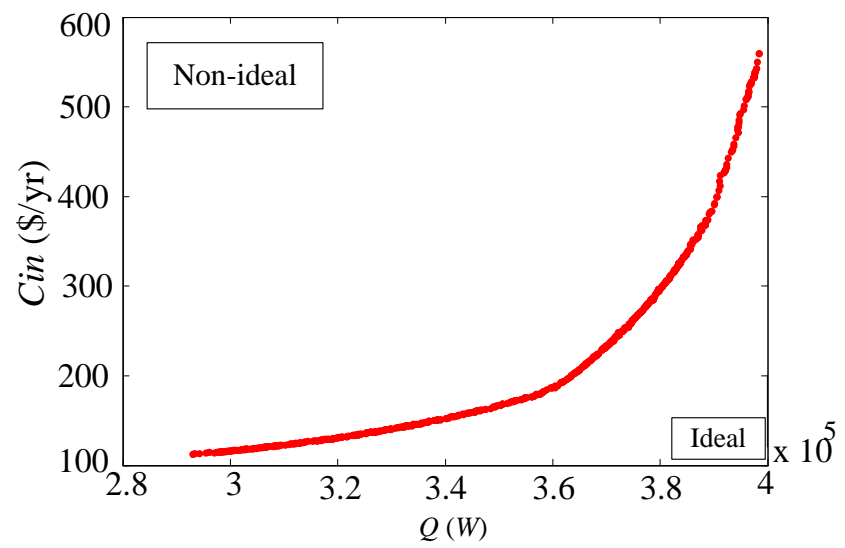

Fig. 6. Rate of heat transfer $(Q)$ vs. $C_{i n}$ Pareto front in the objective space

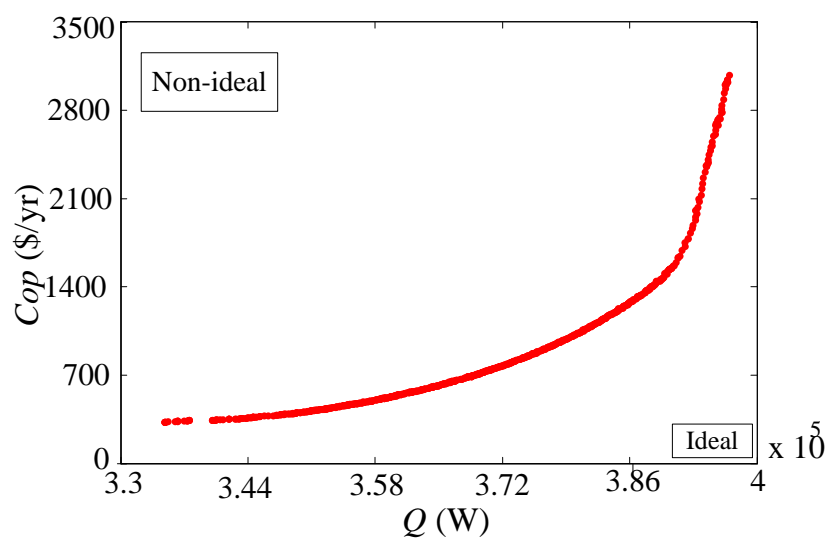

Fig. 7. Rate of heat transfer $(Q)$ vs. $C_{o p}$ Pareto front in the objective space

both $C_{i n}$ and $C_{o p}$ optimization (Fig. 8b). Similar to fin pitch, increase in the fin height decreases (Eq. (14)) the friction factor and hence pressure drop and $C_{o p}$ decreases according to Eqs. (21), (22) and (24). Therefore, $C_{i n}, C_{o p}$ and thus TAC have declining trend. However, with increasing the fin height the number of passages (Eq. (9)) also decreases due to which the trend is different from fin pitch. The trend of $T A C$ closer to the $C_{o p}$ illustrates that the effect of pressure drop on the $T A C$ is much significant.

\subsubsection{Fin offset length}

For fixed lengths of the heat exchanger, increasing the fin offset length $(x)$ has almost no effect on the total heat 


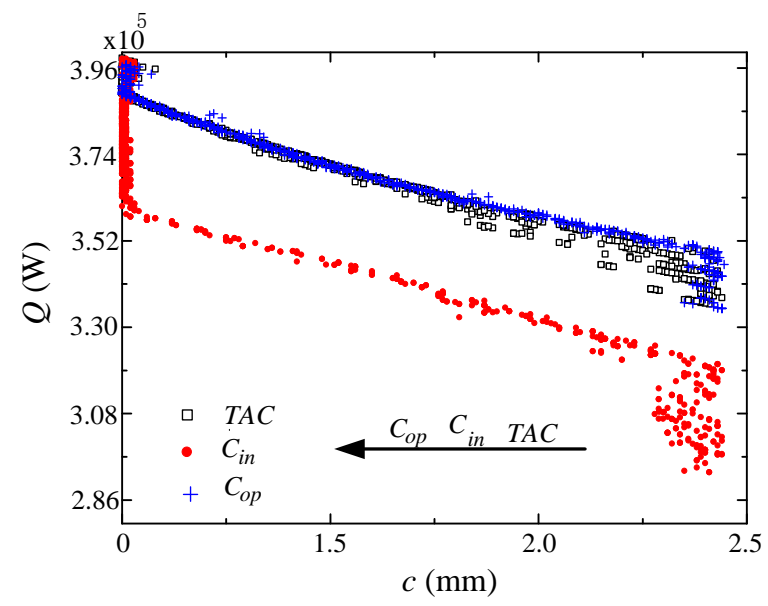

(a)

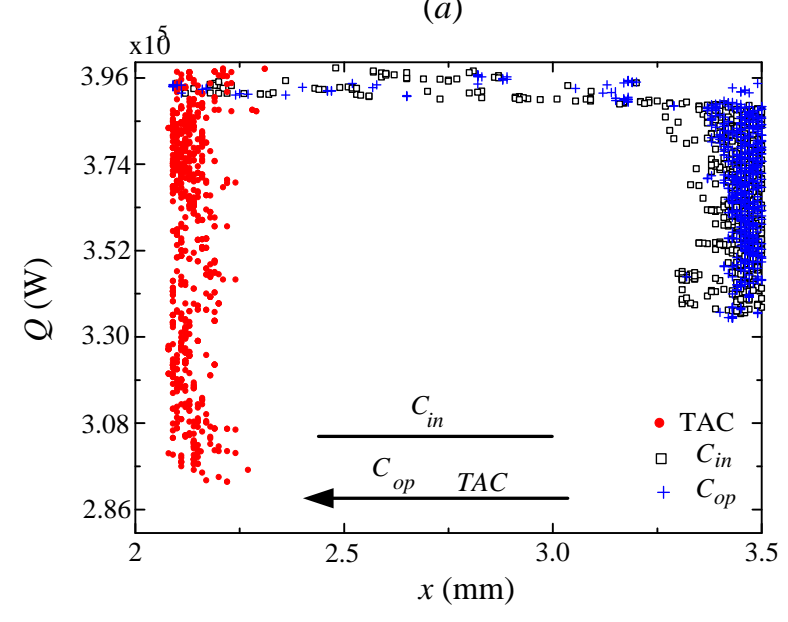

$(c)$

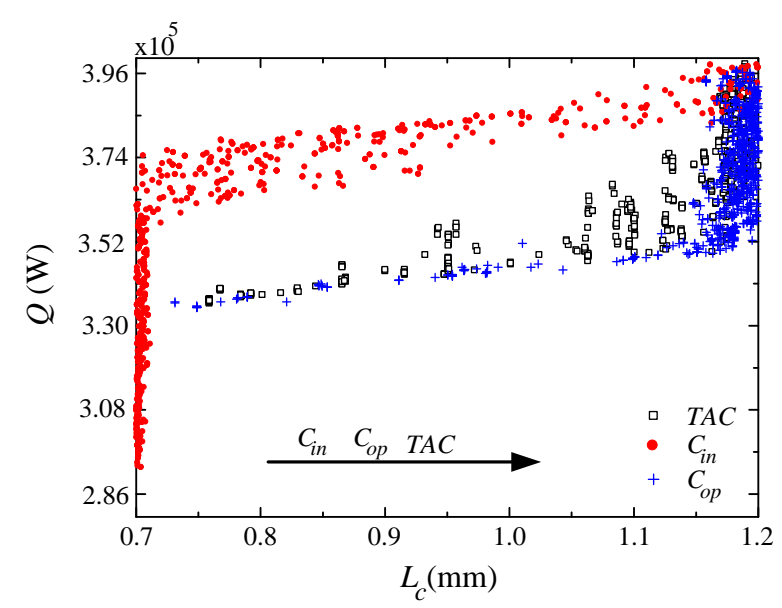

(e)

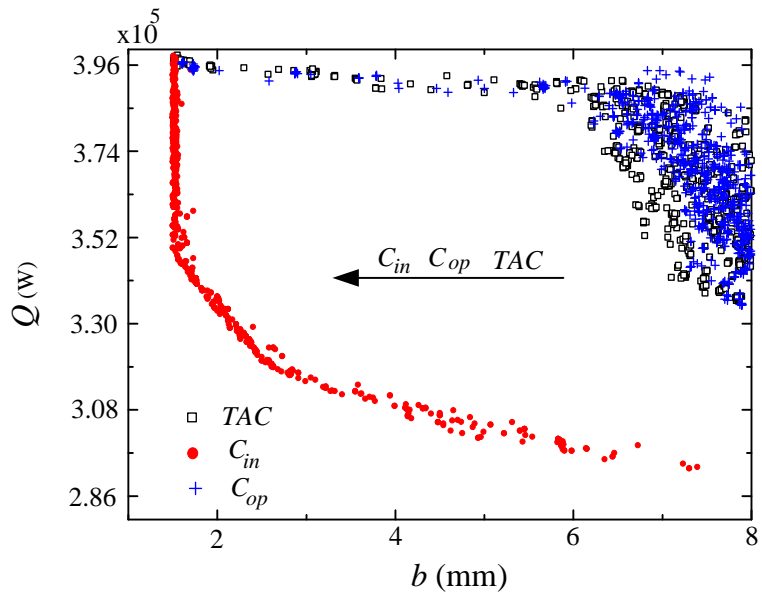

(b)

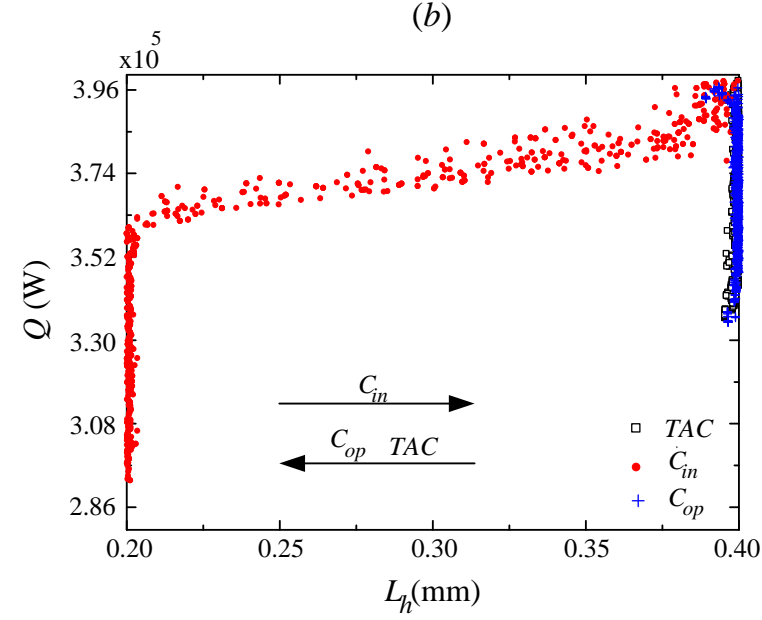

(d)

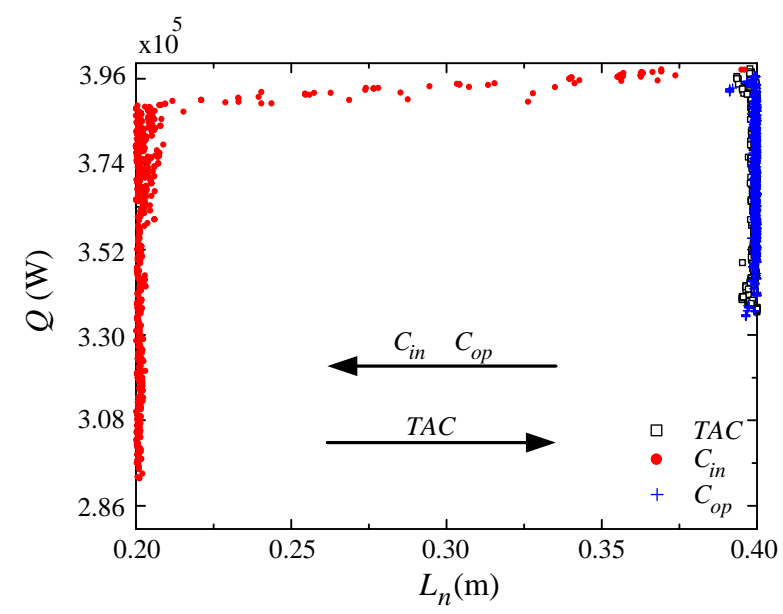

$(f)$

Fig. 8. Distribution of variables for Pareto optimal front, $(a)$ Fin pitch, $(b)$ Fin height, $(c)$ Fin offset length, $(d)$ Hot stream flow length, $(e)$ Cold stream flow length, $(f)$ No-flow length

transfer area due to the fact that the heat transfer surface area per unit volume $(\beta)$ is proportional to $2 / c+2 / b$ and is independent of $x$ (Eq. (6) and $\left.t_{f}<<b, c, x\right)$. However, increasing the offset length decreases the friction factor (Eq. (14)) while no significant effect on $A_{\text {flow }}$ (Eq. (18)) make the pressure drop decreases (Eq. (21)). Hence, $C_{i n}$ is independent of $x$ while $C_{o p}$ and so the TAC covers the whole domain and decreases with increasing offset length as shown in Fig. $8 c$. 


\subsubsection{Hot stream flow length}

Increasing the hot stream flow length $\left(L_{h}\right)$ increases the total heat transfer area (Eq. (5) to (8)) and so the $C_{i n}$. By increasing the heat transfer area, the number of heat transfer units, the effectiveness and thus the rate of heat transfer increases (Eq. (1), (2) and (7). Moreover, increasing the hot stream flow length increases $A_{f l o w, c}$ while has no effect on $A_{\text {flow }, h}$ (Eq. (18) and (5)). Therefore, increasing hot stream flow length increases pressure drop on the hot side due to increase in its heat transfer area, while decreases the pressure drop on the cold side due to decrease in the mass flux (Eq. (17) and (22)) and the $C_{o p}$ decreases. However, in our case due to longer length of the cold side, the decrease in its pressure drop due to decrease in the mass flux is higher than increase in pressure drop on the hot side. Hence, maximum $L_{h}$ is beneficial for lower pressure drop, lower $C_{o p}$ and lower TAC as given in Fig. $8 d$.

\subsubsection{Cold stream flow length}

Increasing the cold stream flow length $\left(L_{c}\right)$ has the same trend for $C_{i n}$ as that of the hot stream flow length which increases the rate of heat transfer. However, due to the smaller length of the hot side the increase in pressure drop on the cold side is higher than decrease on the hot side and hence the pressure drop and the operational cost increases with increasing cold stream flow length (contrary to hot stream flow length) as shown in Fig. $8 e$.

\subsubsection{No-flow length}

By increasing the no-flow length $\left(L_{n}\right)$, the total heat transfer area increases (according to Eqs. (5) to (9)) and thus the investment cost increases. Increasing the heat transfer area increases the $A_{f l o w}$ (Eq. (18)) and decreases the mass flux (Eq. 17). Consequently, the total pressure drop (Eq. (21) and (22)) and the $C_{o p}$ (Eq. (24)) decreases. Furthermore, the rate of heat transfer increases with increasing the heat transfer area (Eq. (1), (2) and (7)). Therefore, the maximum permissible value of no-flow length is required to minimize the $C_{o p}$ and $T A C$.

From above discussion it is concluded that except the hot stream flow length and no-flow length, all other parameters are distributed in its available domain. It illustrates that hot stream flow length and no-flow length has no effect on the conflict between rate of heat transfer $(Q)$ and $T A C$ and they should be at the maximum allowable lengths for the optimum design. In general, the smaller length among hot and cold streams will not contribute in the optimization for equivalent mass flow rates of both streams.

\subsection{Selection of final optimum solution}

In multi-objective optimization problem, it is desirable to select the final optimum solution from the set of nondominated points in the Pareto front. In this study, The Technique for Order of Preference by Similarity to Ideal Solution

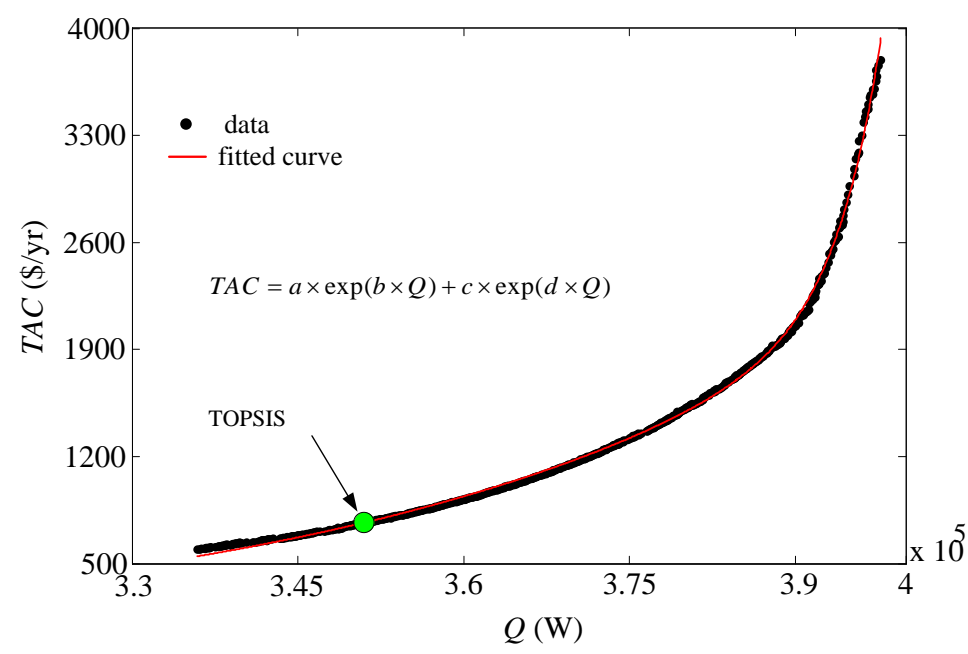

Fig. 9. Fitted curve for $Q$ vs. TAC 


\section{Table 8}

Final optimal values of decision variables with maximum $Q$ and minimum $T A C$

\begin{tabular}{|c|c|c|c|c|c|c|c|c|c|c|}
\hline \multirow[t]{2}{*}{ Method } & \multicolumn{5}{|c|}{ Decision variables } & \multirow[b]{2}{*}{$L_{n}(\mathrm{~m})$} & \multicolumn{4}{|c|}{ Objective functions } \\
\hline & $c(\mathrm{~mm})$ & $\begin{array}{c}b \\
(\mathrm{~mm})\end{array}$ & $\begin{array}{c}x \\
(\mathrm{~mm})\end{array}$ & $L_{h}(\mathrm{~m})$ & $L_{c}(\mathrm{~m})$ & & $C_{o p}$ & $C_{i n}$ & $Q(\mathrm{~W})$ & $\begin{array}{l}\text { TAC } \\
\left(\mathrm{m}^{2}\right)\end{array}$ \\
\hline TOPSIS & 2.27 & 7.93 & 3.5 & 0.4 & 1.1264 & 0.398 & 420.4 & 344.5 & $3.509 \mathrm{E}+05$ & 764.9 \\
\hline
\end{tabular}

(TOPSIS) is introduced to obtain the final solution. The final optimum values of rate of heat transfer $(Q)$ and TAC with the corresponding variables presented in Fig. 9 are listed in Table 8. By comparing these optimum decision variables with Fig. 8, one can observe the significance of their selection. These values are close to the vicinity of abrupt change in rate of heat transfer rate and the TAC (Fig. 8( $a-c$ and $e)$ ). Hence, the TOPSIS provided the best optimal solution between rate of heat transfer and TAC.

Furthermore, the expression for the heat transfer rate as a function of $T A C$ as shown in Fig. 9 is given by:

$$
T A C=1131 \cdot e^{0.3722 Q}+1.163 \cdot e^{4.131 Q}
$$

The $R$ and adjusted $R^{2}$ are approximately equal to 0.988 . The coefficients of fitness in Eq. 26 are within $95 \%$ confidence bounds. The confidence bounds determine the accuracy of these coefficients. The $R^{2}$ is the square of correlation between the mathematical response values and the predicted response values. The value of $R^{2}$ ranges from zero to one where the value closer to one corresponds to better fitness of the model.

\section{Conclusion}

A new method by combining Differential evolution (DE), Genetic Algorithm (GA) and Adaptive Simulated annealing (ASA) algorithms is proposed. The proposed DE-GA-ASA technique linked the three algorithms in the search space and swap population to increase the diversity for finding the global optimum solution. The effectiveness of combined DEGA-ASA method is verified by comparing to some benchmark problems. Then the proposed method is applied for the optimal design of plate fin heat exchanger and its feasibility is approved by comparing with other case studies. Some of the earlier works have either violated one or more constraints. Moreover, their applicability for the multi-objective problems is not investigated. Hence, the present technique is effective for the optimal design of plate-fin heat exchanger. The effect of variation of fin and heat exchanger geometry parameters is also discussed. For this purpose, the heat transfer rate $(Q(\mathrm{~W}))$ and the cost of heat exchanger are considered as objective functions. The design parameters (decision variables) are fin pitch, fin height, fin offset length, cold stream flow length, no-flow length and hot stream flow length. The effect of decision variables on the investment cost and operating cost concluded that the conflict between $Q(\mathrm{~W})$ and $T A C$ are not affected by no-flow length and hot stream flow length. For optimum design, the no-flow length and the smaller flow stream length should be at the maximum allowable lengths when the flow rates are equivalent. Moreover, TOPSIS is introduced for the selection of final optimal solution.

\section{Acknowledgments}

This work is supported by National Science Foundation of China (51210011) and National Science Foundation of Zhejiang Province (LZ13E060001).

\section{Appendices}

In this section, the pseudo codes of DE, GA and ASA are described.

\section{A. Differential evolution algorithm}

Initialize the input values

Initialize all vectors of population randomly

for $i=1$ to $N P$ 
for $j=1$ to $\mathrm{D}$

Initialize gen $=1$

for gen $=1$ to max_gen

for $i=1$ to $N P$

for $m=1$ to $k$

Evaluate the objective functions

\section{Next $m$}

for $j=1$ to $D$

Select 3 different vectors for perturbation

$$
\begin{aligned}
& X_{i, g+1}=X_{r, g}+F_{1}\left(X_{r 1, g}-X_{r 2, g}\right) \\
& u_{j, i, g+1}= \begin{cases}v_{j, i, g+1} & \text { if } \text { rand } \leq C r \vee j=k \\
x_{j, i, g} & \text { otherwise }\end{cases}
\end{aligned}
$$

Start comparing vectors $U_{i, g e n}$ and $X_{i, g e n}$

for $m=1$ to $k$

Evaluate $k_{t h}$ objective function $f_{k}\left(U_{i, g e n}\right)$

$$
\text { If } \begin{gathered}
f_{k}\left(U_{i, g e n}\right)=f_{k}\left(X_{i, g e n}\right) \\
X_{i, g e n+l}=U_{i, g e n}
\end{gathered}
$$

Go to next step

else

$$
\text { Next } m
$$

end

$X_{i, \text { gen }+1}=X_{i, \text { gen }}$

next $m$

next $i$

next gen

Return the best solution

\section{B. Genetic Algorithm}

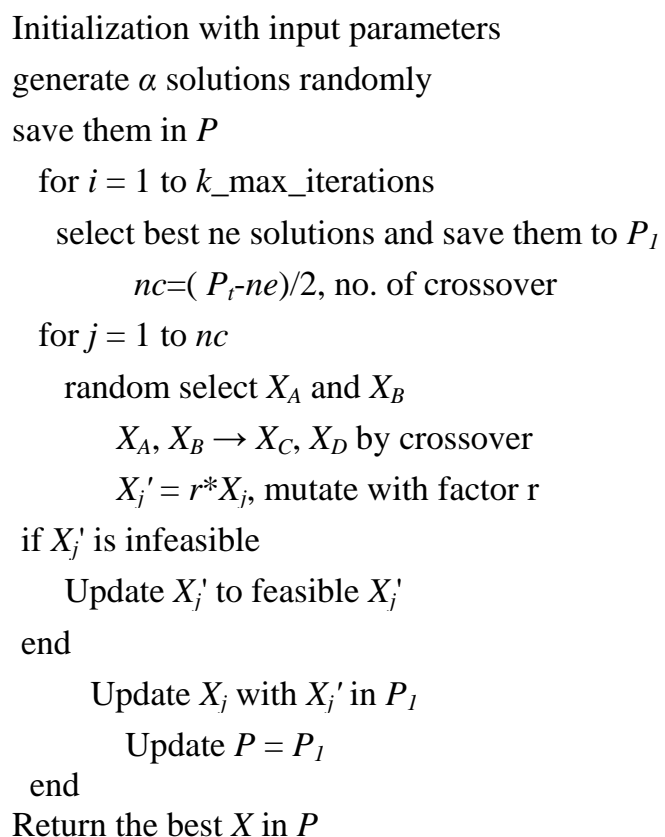




\section{Adaptive simulated annealing}

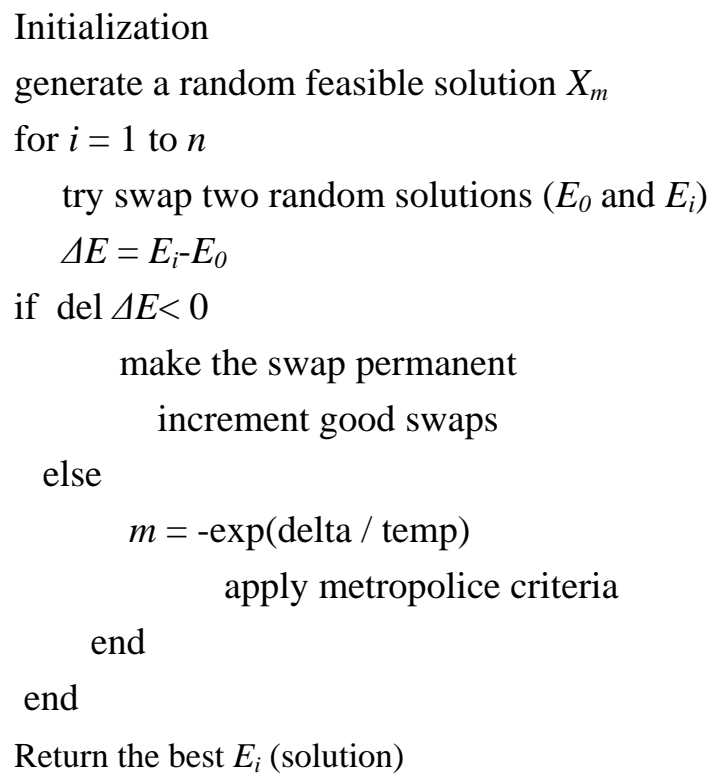

\section{References}

1. Shah, R.K., Advances in science and technology of compact heat exchangers. Heat Transfer Engineering, 2006. 27(5): p. 3-22.

2. Kays, W.M. and A.L. London, Compact heat exchangers. 3rd ed1984, New York: McGraw-Hill. xv, 335 p.

3. Bhowmik, H. and K.S. Lee, Analysis of heat transfer and pressure drop characteristics in an offset strip fin heat exchanger. International Communications in Heat and Mass Transfer, 2009. 36(3): p. 259-263.

4. Wang, L.K. and B. Sunden, Design methodology for multistream plate-fin heat exchangers in heat exchanger networks. Heat Transfer Engineering, 2001. 22(6): p. 3-11.

5. Reneaume, J.M. and N. Niclout, MINLP optimization of Plate Fin Heat Exchangers. Chemical and Biochemical Engineering Quarterly, 2003. 17(1): p. 65-76.

6. Peng, H. and X. Ling, Optimal design approach for the plate-fin heat exchangers using neural networks cooperated with genetic algorithms. Applied Thermal Engineering, 2008. 28(5-6): p. 642-650.

7. Sanaye, S. and H. Hajabdollahi, Thermal-economic multi-objective optimization of plate fin heat exchanger using genetic algorithm. Applied Energy, 2010. 87(6): p. 1893-1902.

8. Xie, G.N., B. Sunden, and Q.W. Wang, Optimization of compact heat exchangers by a genetic algorithm. Applied Thermal Engineering, 2008. 28(8-9): p. 895-906.

9. Najafi, H., B. Najafi, and P. Hoseinpoori, Energy and cost optimization of a plate and fin heat exchanger using genetic algorithm. Applied Thermal Engineering, 2011. 31(10): p. 1839-1847.

10. Wang, Z., Y.Z. Li, and M. Zhao, Experimental investigation on the thermal performance of multi-stream platefin heat exchanger based on genetic algorithm layer pattern design. International Journal of Heat and Mass Transfer, 2015. 82: p. 510-520.

11. Zhao, M. and Y.Z. Li, An effective layer pattern optimization model for multi-stream plate-fin heat exchanger using genetic algorithm. International Journal of Heat and Mass Transfer, 2013. 60: p. 480-489.

12. Chen, C.T. and H.I. Chen, Multi-objective optimization design of plate-fin heat sinks using a direction-based genetic algorithm. Journal of the Taiwan Institute of Chemical Engineers, 2013. 44(2): p. 257-265.

13. Varun and Siddhartha, Thermal performance optimization of a flat plate solar air heater using genetic algorithm. Applied Energy, 2010. 87(5): p. 1793-1799.

14. Yin, H. and R. Ooka, Shape optimization of water-to-water plate-fin heat exchanger using computational fluid dynamics and genetic algorithm. Applied Thermal Engineering, 2015. 80: p. 310-318.

15. Peng, H., X.A. Ling, and E. Wu, An Improved Particle Swarm Algorithm for Optimal Design of Plate-Fin Heat Exchangers. Industrial \& Engineering Chemistry Research, 2010. 49(13): p. 6144-6149.

16. Rao, R.V. and V.K. Patel, Thermodynamic optimization of cross flow plate-fin heat exchanger using a particle swarm optimization algorithm. International Journal of Thermal Sciences, 2010. 49(9): p. 1712-1721. 
17. Yousefi, M. and A.N. Darus, Optimal Design of Plate-fin Heat Exchangers by Particle Swarm Optimization. Fourth International Conference on Machine Vision (Icmv 2011): Computer Vision and Image Analysis: Pattern Recognition and Basic Technologies, 2012.8350.

18. Yousefi, M., R. Enayatifar, and A.N. Darus, Abdullah, A. H., Optimization of plate-fin heat exchangers by an improved harmony search algorithm. Applied Thermal Engineering, 2013. 50(1): p. 877-885.

19. Yousefi, M., R. Enayatifar, and A.N. Darus, Optimal design of plate-fin heat exchangers by a hybrid evolutionary algorithm. International Communications in Heat and Mass Transfer, 2012. 39(2): p. 258-263.

20. Zarea, H., Kashkooli, F. M., Mehryan, A. M., Saffarian, M. R., and E.N. Beherghani, Optimal design of platefin heat exchangers by a Bees Algorithm. Applied Thermal Engineering, 2014. 69(1-2): p. 267-277.

21. Hadidi, A., A robust approach for optimal design of plate fin heat exchangers using biogeography based optimization (BBO) algorithm. Applied Energy, 2015. 150: p. 196-210.

22. Hajabdollahi, H., M. Tahani, and M.H.S. Fard, CFD modeling and multi-objective optimization of compact heat exchanger using CAN method. Applied Thermal Engineering, 2011. 31(14-15): p. 2597-2604.

23. Wen, J., Yang, H. Z., Tong, X., Li, K., Wang, S. M., and Y.Z. Li, Optimization investigation on configuration parameters of serrated fin in plate-fin heat exchanger using genetic algorithm. International Journal of Thermal Sciences, 2016. 101: p. 116-125.

24. Zheng, J., R. Shen, and J. Zou, Enhancing diversity for NSGA-II in evolutionary multi-objective optimization, in Natural Computation (ICNC), 2012 Eighth International Conference on 2012 p. 4.

25. Sun, Y.J. and G.Z. Shen, Improved NSGA-II Multi-objective Genetic Algorithm Based on Hybridizationencouraged Mechanism. Chinese Journal of Aeronautics, 2008. 21(6): p. 540-549.

26. Muralikrishna, K. and U.V. Shenoy, Heat exchanger design targets for minimum area and cost. Chemical Engineering Research \& Design, 2000. 78(A2): p. 161-167.

27. Jia, R. and B. Sundén. Optimal Design of Compact Heat Exchangers by an Artificial Neural Network Method. in ASME 2003 Heat Transfer Summer Conference. 2003. Las Vegas, Nevada, USA: Heat Transfer Division.

28. Coello, C.A.C., G.B. Lamont, and D.A.V. Veldhuizen, Evolutionary Algorithms for Solving Multi-Objective Problems. Second Edition ed. Genetic and Evolutionary Computation Series, ed. D.E. Goldberg2007, New York, NY 10013, USA: Springer US

29. Gujarathi, A.M. and B.V. Babu, Differential Evolution Strategies for Multi-objective Optimization. Proceedings of the International Conference on Soft Computing for Problem Solving (Socpros 2011), Vol 1, 2012. 130: p. 63-71.

30. Babu, B.V. and M.M.L. Jehan, Differential evolution for multi-objective optimization. Cec: 2003 Congress on Evolutionary Computation, Vols 1-4, Proceedings, 2003: p. 2696-2703.

31. Fonseca, C.M. and P.J. Fleming. Genetic Algorithms for Multiobjective Optimization: FormulationDiscussion and Generalization. in Proceedings of the 5th International Conference on Genetic Algorithms. 1993. San Francisco, CA, USA: Morgan Kaufmann Publishers Inc.

32. Konak, A., D.W. Coit, and A.E. Smith, Multi-objective optimization using genetic algorithms: A tutorial. Reliability Engineering \& System Safety, 2006. 91(9): p. 992-1007.

33. Perumal, A.I. and S.P. Rajagopalan, Adaptive Simulated Annealing-Useful Lessons Learned. International journal of soft computing, 2007. 2(5): p. 572-579.

34. Incropera, F.P., Fundamentals of heat and mass transfer. 6th ed2007, Hoboken, NJ: John Wiley. xxv, 997 p.

35. Manglik, R.M. and A.E. Bergles, Heat-Transfer and Pressure-Drop Correlations for the Rectangular Offset Strip Fin Compact Heat-Exchanger. Experimental Thermal and Fluid Science, 1995. 10(2): p. 171-180.

36. Deb, K., Multi-objective optimization using evolutionary algorithms. 1st ed. Wiley-Interscience series in systems and optimization2001, Chichester ; New York: John Wiley \& Sons. xix, 497 p.

37. Storn, R., System design by constraint adaptation and differential evolution. IEEE Transactions on Evolutionary Computation, 1999. 3(1): p. 22-34.

38. Radha, T., P. Millie, and A. Ajith, A simple adaptive Differential Evolution algorithm, in Nature \& Biologically Inspired Computing, 2009. NaBIC 2009. World Congress on 2009, IEEE

39. Holland, J., Adoption in Natural and Artificial System, 1992, MIT Press, Cambridge, MA.

40. Kirkpatrick, S., C.D.J. Gelatt, and M. Vecchi, Optimization by simulated annealing. Science, 1983. 220(4598): p. 671-680.

41. Wolpert, D.H. and W.G. Macready, No free lunch theorems for optimization. IEEE Transactions on Evolutionary Computation, 1997. 1(1): p. 67-82.

42. Bin, X., C. Jie, and Z. Juan, Hao, F., Zhi-Hong, P., Hybridizing Differential Evolution and Particle Swarm Optimization to Design Powerful Optimizers: A Review and Taxonomy. IEEE Transactions on Systems, Man, and Cybernetics, Part C: Applications and Reviews, 2012. 42(5): p. 744-767.

43. Chen, S.-J.J. and C.L. Hwang, Fuzzy Multiple Attribute Decision Making: Methods and Applications 1992, New York, Secaucus, NJ, USA: Springer-Verlag New York, Inc. Secaucus, NJ, USA, 1992.

44. Deb, K., Pratap, A., Agarwal, S., and T. Meyarivan, A fast and elitist multiobjective genetic algorithm: NSGA-II. IEEE Transactions on Evolutionary Computation, 2002. 6(2): p. 182-197. 